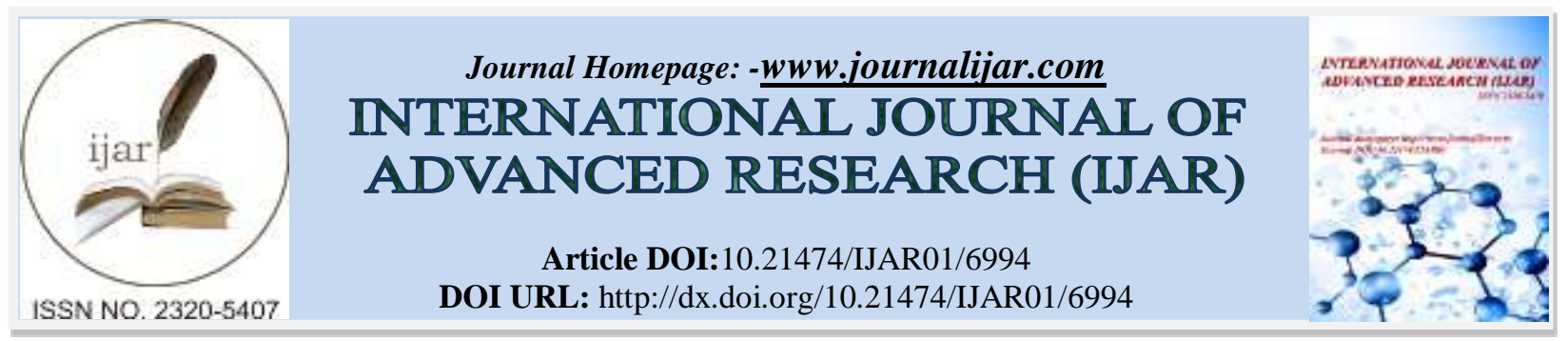

RESEARCH ARTICLE

\title{
TO STUDY INDIVIDUAL AND COMBINED ANTIBIOTICS EFFICACY AGAINST RESISTANT PATHOGEN ISOLATED FROM DIABETIC FOOT INFECTION.
}

Patil Y.S.

Shri. Dnyaneshwar Maskuji Burungale Science College, Shegaon. Dist. Buldana (MS).

\section{Manuscript Info}

Manuscript History

Received: 19 February 2018

Final Accepted: 21 March 2018

Published: April 2018

\section{Keywords:-}

Diabetic foot infections, Antibiotics, antibiotic resistance, Antibiotic combination, Synergy.

\section{Abstract}

Diabetic foot infections typically begin in a wound, most often due to neuropathic ulceration. Diabetic foot infection facing unique challenge of successful treatment because of wide spread of resistant bacteria to many antibiotics. Combination antibiotic therapy could be helpful to combat these resistant bacteria. This basically depends on the microbial etiology and selection of appropriate antibiotics for combination therapy. Using manual E-test method, Minimum inhibitory concentration (MIC) was determined susceptibility to individual antibiotics. Fractional inhibitory concentration index (FICI) was used to determine synergistic effects for combined antibiotics. . Gentamicin susceptibility showed 100\%, 20\%,30\%,80\% and 70\% of Escherichia coli, Klebsiella pneumonia, Proteus spp, Pseudomonas spp, and Staphylococcus aureus, were sensitive, respectively. Ciprofloxacine showed $80 \%, 30 \%, 60 \%, 40 \%$ and $90 \%$ resistant to Escherichia coli, Klebsiella pneumonia, Proteus spp, Pseudomonas spp, and Staphylococcus aureus, respectively. Ceftriaxone showed 50\%, 40\% 60\%, 10\%, and 70\% resistant result against Escherichia coli, Klebsiella pneumonia, Proteus spp, Pseudomonas spp, and Staporhylococcus aureus, respectively. Fractional inhibitory concentration index (FICI) recorded and synergistic effects were determined. Knowledge on the antibiotic synergy pattern of the isolates will be helpful in determining the drugs for the empirical treatment of diabetic foot infections.

Copy Right, IJAR, 2018,. All rights reserved.

\section{Introduction:-}

Foot infections in individuals with diabetes are an increasingly common public health problem and are associated with mortality and morbidity. Diabetes mellitus is a metabolic syndrome characterized by hyperglycemia due to defects in insulin secretion, function. Diabetic patients are more susceptible to infections; in particular foot infections which are a common and serious problem and sometimes lead to complications and amputations. Diabetic patients have a lifetime risk as high as $25 \%$ for developing foot ulceration (Singh N, et al; 2005). It is characterized by several pathological complications such as neuropathy, peripheral vascular disease, foot ulceration and infection with or without osteomyelitis, which leads to the development of gangrene and which even necessitates limb amputation. (Baron E J, et al; 2011) The individuals with diabetes have at least a 10-fold greater risk of being hospitalized for soft tissue and bone infections of the foot than individuals without diabetes. (Bowler P G, et al; 2011) The impaired micro-vascular circulation in patients with diabetic foot limits the access of phagocytes 
favouring development of infection (Anandi C,et al; 2004, Gadepalli R, et al; 2006). The local injuries and the improper foot wear further compromise the blood supply in the lower extremities. While the foot infections in persons with diabetes are initially treated empirically, a therapy which is directed at the known causative organisms may improve the outcome. (Amitabha B.,2012) Antimicrobial resistance is an increasing global public health problem affecting both developed and resource-limited countries; and prompted call for action worldwide (DiazGranados,C.A.et al;2008). Selection of appropriate antibiotic for management of diabetic foot infection is crucial and largely dependent on identification of microbial pathogen and evaluation of antibiotic susceptibility (Crouzet, J.et al; 2011). Escherichia coli, Proteus spp., Pseudomonas spp., Staphylococcus aureus and Enterococcus spp. are the most frequent pathogens contributing to progressive and widespread tissue destruction. (Anandi C,et al; 2004, Gadepalli R, et al; 2006). Diabetic foot infections are often polymicrobial (Alavi S.M.et al; 2007 and Gadepalli R, et al; 2006). Appropriate selection of antibiotics based on the antibiograms of the isolates from the lesions is most critical for the proper management of these infections. Nevertheless, the initial empirical therapy is often decided based on the knowledge of the susceptibility profile of the prevalent microbial flora recovered from the previous cases. The use of two antibiotics prevents the emergence of resistance to either of the antibiotics if used separately as a monotherapy (Leekha, S.et al; 2011).

The purpose of this study was to define the range of resistance of bacteria isolated from diabetic foot infections and further evaluate synergistic effect of combination antibiotics to guide proper therapy.

\section{Materials and Methods:-}

This study included twenty-five individuals with diabetic foot infection at multispeciality hospital Akola, March and April of 2016. Laboratory analysis was conducted in department of Microbiology, Shegaon. The study focused on evaluation of the five most prevalent bacteria isolated from diabetic foot infections, which include Escherichia coli, Klebsiella pneumonia, Proteus spp, Pseudomonas spp, and Staphylococcus aureus. five isolates of each isolated pathogen were evaluated for sensitivity testing.

\section{Materials:-}

Antibiotics used in study: Commercial antibiotics standard solution accompanied by a statement of its activity in $\mathrm{mg} / \mathrm{ml}$ for Gentamicin (Hi-media, India), Ciprophloxacin (Hi-media, India), and Ceftriaxone antibiotics (Hi-media, India), collected from the Hi-media seller in Amravati. Isolates were tested against gentamicin, ciprofloxacin, ceftriaxzone, then against a combination of gentamicin with ciprofloxacin and combination of gentamicin with ceftriaxzone.

\section{Methods:- \\ E-test Method:-}

Two fold dilutions of the antibiotic solution in sterile normal saline were prepared for each individual antibiotic (Table 1). This was followed by impregnation via the immersion method; blank sterile disks were soaked in the different concentration of each antibiotic for half an hour. Then the impregnated disks were transferred into sterile Petri dishes. These were allowed to dry and stored in a freezer at $-14^{\circ} \mathrm{C}$.

\section{The Antibiotic Susceptibility Test for isolates:-}

The strains of bacterial isolates were collected from infected foot of diabetic patients. Isolated strains were homogenized in $0.85 \% \mathrm{NaCl}$ to achieve $0.5 \mathrm{McFarland}$ 's turbidity. A sterile, non-toxic swab was dipped into the inoculums suspension and pressed against the inside of the tube. Then, the surface of Muller Hinton agar in petridish was inoculated with suspension. Sterile forceps were used to deposit the antibiotic discs onto the surface of the inoculated media. Disks were made in complete contact with the agar surface, which was located in one line beginning with the highest concentration to the lowest one of the same antibiotics. The plate was then incubated at $37^{\circ} \mathrm{C}$ for 24 hours. After incubation, an elliptical zone of inhibition was produced and the point at which the ellipse met the defined disk concentration represented the minimum inhibitory concentration (MIC) of the antibiotics according to Clinical laboratory Standards Institute (CLSI 2013). Fractional inhibitory concentration index (FICI) was used to interpret the result. The FICs were calculated as follows: $\Sigma F I C=F I C(A)+F I C ~(B)$; where FIC (A) is the MIC of drug (A) in combination / MIC of drug (A) alone. And FIC (B) is the MIC of drug B in the combination/MIC of drug (B) alone (Meletiadis, J.et al; 2010). 
Table 1:-Different gradient concentration of antibiotics $(\mathrm{mg} / \mathrm{ml})$ dilution

\begin{tabular}{|c|c|c|c|c|c|c|c|c|}
\hline \multirow{2}{*}{ Sr.no. } & Antibiotic (mg/mL) & \multicolumn{7}{|c|}{ Tubes 1-8 } \\
\cline { 3 - 9 } & & $\begin{array}{c}1 \\
\mathrm{mg} / \mathrm{ml}\end{array}$ & $\begin{array}{c}2 \\
\mathrm{mg} / \mathrm{ml}\end{array}$ & $\begin{array}{c}3 \\
\mathrm{mg} / \mathrm{ml}\end{array}$ & $\begin{array}{c}4 \\
\mathrm{mg} / \mathrm{ml}\end{array}$ & $\begin{array}{c}5 \\
\mathrm{mg} / \mathrm{ml}\end{array}$ & $6 \mathrm{mg} / \mathrm{ml}$ & $7 \mathrm{mg} / \mathrm{ml}$ \\
\hline 1 & Gentamicin & 80 & 40 & 20 & 10 & 5 & 2.5 & 1.25 \\
\hline 2 & Ciprophloxacin & 200 & 100 & 50 & 25 & 12.5 & 6.25 & 3.16 \\
\hline 3 & Ceftriaxzone & 250 & 125 & 62.5 & 31.25 & 15.63 & 7.81 & 3.91 \\
\hline 4 & Gentamicin/Ciprofloxacin & $80 / 200$ & $40 / 100$ & $20 / 50$ & $10 / 25$ & $5 / 12.5$ & $2.5 / 6.25$ & $1.25 / 3.16$ \\
\hline 5 & Gentamicin/Ceftriaxone & $80 / 250$ & $40 / 125$ & $20 / 62.5$ & $10 / 31.25$ & $5 / 15.63$ & $2.5 / 7.81$ & $1.25 / 3.91$ \\
\hline 6 & Gentamicin & 80 & 40 & 20 & 10 & 5 & 2.5 & 1.25 \\
\hline
\end{tabular}

\section{Results:-}

The results showed that $100 \%$ of the E. coli strains were sensitive to gentamicin with (MIC $=6.6 \mathrm{mg} / \mathrm{ml}$ ), $70 \%$ of Staphylococcus aureus were sensitive $(\mathrm{MIC}=0.6 \mathrm{mg} / \mathrm{ml})$, only $20 \%$ were resistant $(\mathrm{MIC}=20-80 \mathrm{mg} / \mathrm{ml})$. Klebsiella pneumoniae were sensitive to $20 \%$ only (MIC=20-80mg/ml). Protus spp showed that $30 \%$ were sensitive $(\mathrm{MIC}=0.6-1.25 \mathrm{mg} / \mathrm{ml}), 20 \%$ were intermediate $(\mathrm{MIC}=10 \mathrm{mg} / \mathrm{ml})$ and $50 \%$ were resistant $(\mathrm{MIC}=20-80 \mathrm{mg} / \mathrm{ml})$. Pseudomonas aureginosa, showed that $20 \%$ were resistant $(\mathrm{MIC}=40-80 \mathrm{mg} / \mathrm{ml}$ ) and $80 \%$ were sensitive (MIC $=0.6$ $2.5 \mathrm{mg} / \mathrm{ml}$ ). for ciprofloxacin, only $10 \%$ of Pseudomonas aureginosa were sensitive, while the other strains showed high rate of resistant $80 \%$ for E.coli with $\mathrm{MIC}=25-200 \mathrm{mg} / \mathrm{ml}$, Klebsiella pneumoniae were resistant to $30 \%$ only (MIC $=20-80 \mathrm{mg} / \mathrm{ml}), 60 \%$ for Protus.spp with $\mathrm{MIC}=25-100 \mathrm{mg} / \mathrm{ml}$ and $40 \%$ for Pseudomonas aureginosa with MIC $=6.25-200 \mathrm{mg} / \mathrm{ml}$. However, $10 \%$ of Staphylococcus aureus were of intermediate sensitivity (MIC=1.6mg $/ \mathrm{ml})$. For ceftriaxone, it is clear that the four strains showed closed intermediate susceptibility result. While S.aureus was sensitive $(30 \%)$ with $\mathrm{MIC}=1.9-7.8 \mathrm{mg} / \mathrm{ml}$ and Protus.spp was highly resistant $(60 \%)$ with MIC=7.8-62.5mg/ml (Table 2). The combination of gentamicin/ciprofloxacin, gentamicin/ ceftriaxone, produced the lowest mean FIC value for Pseudomonas aureginosa with 20\%-40\% of combinations demonstrating synergy. In contrast, in E coli the highest antagonism effect (100\%). On the other hand, Staphylococcus aureus, Klebsiella pneumoniae and Protus spp gave highly indifferent effect (80\%-90\%) (70\%-80\%), respectively (Fig. 1, 2).

Table 2:-Sensitivity pattern of Gentamicin, Ciprofloxacin and ceftriaxone individually on the five most common bacterial isolates from diabetic foot infection.

\begin{tabular}{|c|l|c|c|c|c|c|c|c|c|c|c|}
\hline Sr.no. & \multirow{2}{*}{ Organisms } & \multicolumn{3}{|c|}{ gentamicin } & \multicolumn{3}{c|}{ ciprofloxacin } & \multicolumn{3}{c|}{ ceftriaxone } \\
\cline { 3 - 11 } & & $\mathrm{S} \%$ & $\mathrm{I} \%$ & $\mathrm{R} \%$ & $\mathrm{~S} \%$ & $\mathrm{I} \%$ & $\mathrm{R} \%$ & $\mathrm{~S} \%$ & $\mathrm{I} \%$ & $\mathrm{R} \%$ \\
\hline 1 & E.coli & 100 & 0 & 0 & 0 & 20 & 80 & 0 & 50 & 50 \\
\hline 2 & Klebsiella pneumoniae & 20 & 20 & 60 & 0 & 70 & 30 & 10 & 50 & 40 \\
\hline 3 & Protus spp & 30 & 20 & 50 & 10 & 30 & 60 & 0 & 40 & 60 \\
\hline 4 & Pseudomonas aureginosa & 80 & 0 & 20 & 0 & 60 & 40 & 70 & 20 & 10 \\
\hline 5 & Staphylococcus aureus & 70 & 10 & 20 & 0 & 10 & 90 & 0 & 30 & 70 \\
\hline
\end{tabular}

S: sensitive, I: intermediate, R: resistance

\section{Discussion:-}

Antimicrobial resistance is an increasingly serious threat to global public heath that needs urgent action. There are many factors contribute to the emergence of antimicrobial resistance and its spread in the community. These include exposure of patients to MDR bacteria while receiving medical care in healthcare facilities, inappropriate antibiotics use and lack of antimicrobial stewardship efforts, inadequate infection control measures, travel of people and goods, socioeconomic factors, antibiotic residues in the environment, bacterial gene transfer and clonal spread (Levy, S. B. 2002). Our study showed that E.coli was highly sensitive to gentamicin (100\%) while it was $80 \%$ resistant to ciprofloxacin. This result is similar to the finding of Kibret and Abera (Kibret, M. \& Abera, B. 2011). who reported $79.6 \%$ of E.coli sensitivity to gentamicin, while recording high degree of sensitivity to ciprofloxacin. Regarding ceftriaxone, the result showed zero percent sensitivity, thus similar to study done in South Africa that tested resistant to ceftriaxone (Lowman, W. et al; 2012). Pseudomonas spp showed 70\% sensitivity rate to ceftriaxone which is less than other study which gave $92.7 \%$ (Masood, S. H. and Aslam, N. 2010). Gentamicin in this study inhibited 20\% of Pseudomonas strains comparing with other study which recorded higher inhibition rate (87.5\%) (Anguzu, J. R. and Olila, D. 2007). Ciprofloxacin showed high resistant rate this is similar to the study done in Saudi Arabia (Akhtar, N.et al; 2010). Proteus spp showed $60 \%$ resistant to both ceftriaxone and ciprofloxacin which greatly agrees with that $83.8 \%$ sensitively reported by (Masood et.al;.2010). 30\% of Proteus.spp was sensitive to gentamicin comparing 
with 36.8\% sensitivity recorded by Saleh and Hatem (Bahashwan,et al; 2013). Staphylococcus aurous showed 30\% sensitivity to ceftriaxone. This result agrees with the study of Emmanuel and Magaji ( Nwankwo,et al; 2011) who reported $71.8 \%$ sensitive. While S.aureus was resistant to ciprofloxacin, gentamicin 90\%, 20\%, respectively. This result is almost similar with the studies of Mounir and Bekhit (Salem-bekhit,et al; 2014) who reported 48.7\% and $14 \%$ of S. aureus colonies resistant to ciprofloxacin and gentmicin, respectively. The resistance of these bacteria to antibiotics can be partly attributed to the ability of these bacteria to form small colony variants following the exposure to antibiotics or environmental stressors such as cold stress (Samuelsen, et al; 2005) (Onyango,et al;2012). Combination antibiotic treatments can have significant effects on bacterial survival. Therefore, two combinations of antibiotics can be more effective in treatment of infections. The synergistic effects were observed in $20 \%$ of Pseudomonas.spp isolates to gentamicin and ciprofloxacin combination, our finding replicates the study of (Yasmin et al; 2013) who observed synergy in $16.7 \%$ of isolates. Similarly, combination of gentamicin /ceftriaxone showed $40 \%$ synergy effect, in agreement with the study of (Angehrn 1983). who recorded 67.5\% synergy. Ciprofloxacin /gentamicin combination showed antagonistic effect against E.coli, this result disagrees with the study of (Dickgiesser, et al; 1986) who reported synergistic effect. The same authors reported indifferent effect at the combination agent to S.aureus this agrees with the present result. Proteus spp demonstrated indifferent effect to gentamicine/ciprofloxacin combination. This result disagrees with the study of( Weiss, et al;1986) who reported higher susceptibility of proteus spp when subjected to gentamicin/ciprofloxacin combination. Access to microbiology services for identification of microbial etiology and antimicrobial sensitivity profiles is crucial to guide effective optimal antimicrobial treatment; emphasizing the importance of adoption of quality assurance measures for microbiology laboratories. Antimicrobial susceptibilities including combination drugs and their interaction would inform appropriate therapy and minimize the emergence of antimicrobial resistance.

\section{Conclusions:-}

This study attempted to evaluate the susceptibility results of individual and synergistic effect of two combination antibiotics, gentamicin plus ciprofloxacin and gentamicin plus ceftriaxone, using manual E- test method. The individual antibiotics displayed resistance pattern to ciprofloxacin and ceftriaxone against the five isolated strains. The gentamicin antibiotic displayed synergistic effect when combined with ciprofloxacin and with ceftriaxone against Pseudomonas aeruginosa, but it had antagonistic effect when tested against E.coli. The other three organisms, Staphylococcus aureus, Klebsiella pneumoniae and Proteus spp, showed indifferent effect. Combination antibiotics strategy should be considered to target bacterial pathogens with emerging resistance pattern. The synergistic and antagonistic effects among the different antibiotics combinations to maximize the efficacy of antimicrobials and prevent emergence of bacterial resistance.

\section{References:-}

1. Akhtar, N., Alqurashi, A. M. and Abu Twibah, M. (2010). In vitro ciprofloxacin resistance profiles among gram-negative bacteria isolated from clinical specimens in a teaching hospital. J. Pak. Med. Assoc. 60, 625-7 (2010).

2. Alavi SM, Khosravi AD, Sarami A, Dashtebozorg A, Montazeri EA. Bacteriologic study of diabetic foot ulcer. Pak J Med Sci 2007; 23: 681-4.

3. Amitabha Bhattacharjee. Antimicrobial susceptibility of pseudomonas aeruginosa isolated from wound infections, Indian journal of dermatology. 2012, vol 51(4), 286-288.

4. Anandi C, Alaguraja D, Natarajan V, Ramanathan M, Subramaniam CS, Thulasiram M, et al. Bacteriology of diabetic foot lesions. Indian Med Microbiol 2004; 22: 175-8.

5. Angehrn, P. (1983). In vitro and in vivo synergy between ceftriaxone and aminoglycosides against Pseudomonas aeruginosa. Eur. J. Clin. Microbiol. 2, 489-95 (1983).

6. Anguzu, J. R. and Olila, D. (2007). Drug sensitivity patterns of bacterial isolates from septic post-operative wounds in a regional referral hospital in Uganda. Afr. Health Sci. 7, 148-54 (2007).

7. Bahashwan, S. A. \& Shafey, H. M. El. (2013). Antimicrobial resistance patterns of proteus isolates from clinical specimens. Eur. Sci. J. 9, 1857-788 (2013).

8. Baron E J, Thomson RB. Specimen collection, transport and processing: bacteriology .manual of clinical microbiology. 2011, vol (10), 228-271.

9. Bowler P G, Duerden B I, Armstrong DG .wound microbiology and associated approaches to wound management. Clin Microbiol. 2011, vol (14), 244-269. 
10. Clsi. (2013). Performance Standards for Antimicrobial Susceptibility Testing; Twenty-Second Informational Supplement. Clinical and Laboratory Standards Institute 32, (2013).

11. Crouzet, J., Lavigne, J. P., Richard, J. L. and Sotto, A (2011). Diabetic foot infection: a critical review of recent randomized clinical trials on antibiotic therapy. Int. J. Infect. Dis. 15, e601-10 (2011).

12. DiazGranados, C. A., Cardo, D. M. and McGowan, J. E. (2008). Antimicrobial resistance: international control strategies, with a focus on limited-resource settings. International Journal of Antimicrobial Agents 32, 1-9 (2008).

13. Dickgiesser, N., in der Stroth, S. and Wundt, W. (1986). Synergism of ciprofloxacin with beta-lactam antibiotics, gentamicin, minocycline and pipemidic acid]. Infection 14, 82-5 (1986).

14. Gadepalli R, Dhawan B, Sreenivas V, Kapil A, Ammini AC, Chaudhry R.A clinico-microbiological study of diabetic foot ulcers in an Indian tertiary care hospital. Diabetes Care 2006; 29: 1727-32.

15. Kibret, M. \& Abera, B. (2011). Antimicrobial susceptibility patterns of E. coli from clinical sources in northeast Ethiopia. Afr. Health Sci. 11 Suppl 1, S40-5 (2011).

16. Leekha, S., Terrell, C. L. \& Edson, R. S. (2011). General principles of antimicrobial therapy. Mayo Clin. Proc. 86, 156-67 (2011).

17. Levy, S. B. (2002). Factors impacting on the problem of antibiotic resistance. J. Antimicrob. Chemother. 49, 25-30 (2002).

18. Lowman, W., Aithma, N., Duse, A. G. and Mer, M. (2012). Comparative MIC evaluation of a generic ceftriaxone by both microdilution on clinically relevant isolates from an academic hospital complex in South Africa. South African Medical Journal 102, 102-103 (2012).

19. Masood, S. H. and Aslam, N. (2010). In Vitro Susceptibility Test of Different Clinical Isolates against Ceftriaxone. Oman Med. J. 25, 199-202 (2010).

20. Meletiadis, J., Pournaras, S., Roilides, E. and Walsh, T. J. (2010). Defining fractional inhibitory concentration index cutoffs for additive interactions based on self-drug additive combinations, Monte Carlo simulation analysis, and in vitro-in vivo correlation data for antifungal drug combinations against Aspergillus fumi. Antimicrob. Agents Chemother. 54, 602-9 (2010).

21. Nwankwo, E. O. and Nasiru, M. S. (2011). Antibiotic sensitivity pattern of Staphylococcus aureus from clinical isolates in a tertiary health institution in Kano, Northwestern Nigeria. Pan Afr. Med. J. 8, 4 (2011).

22. Onyango LA, Dunstan RH, Gottfries J, von Eiff C, Roberts TK. (2012). Effect of Low Temperature on Growth and

23. Salem-bekhit, M. M. Phenotypic and Genotypic Characterization of Nosocomial Isolates of Staphylococcus aureus with Reference to Methicillin Resistance. 13, 1239-1246 (2014).

24. Samuelsen O, Haukland HH, Kahl BC, von Eiff C, Proctor RA, Ulvatne H, et al. (2005). Staphylococcus aureus small colony variants are resistant to the antimicrobial peptide lactoferricin B. J Antimicrob Chemother. 2005;56(6):1126-9.

25. Singh N, Armstrong DG, Lipsky BA. Preventing foot ulcers in patients with diabetes. JAMA 2005; 293: 217 28.

26. Ultra-Structure of Staphylococcus spp. PLoS One. 2012; 7(1): e29031. PubMed Central PMCID: PMC3265459.

27. Weiss, D., Trautmann, M., Wagner, J., Borner, K. and Hahn, H. (1986). Ciprofloxacin: a comparative evaluation of its bactericidal activity in human serum against four enterobacterial species. Drugs Exp. Clin. Res. 12, 889-94 (1986).

28. Yasmin, F., Akhtar, N. and Hameed, A. (2013). Report: In vitro synergistic Effect of Ciprofloxacin with Aminoglycosides against Multidrug resistant-Pseudomonas aeruginosa. Pak. J. Pharm. Sci. 26, 1041-4 (2013). 